\title{
Cilostazol Use Is Associated with Reduced Risk of Dementia: A Nationwide Cohort Study
}

\author{
Shu-Yu Tai ${ }^{1,2,3,4} \cdot$ Chen-Yu Chien $^{5,6,7} \cdot$ Yu-Han Chang ${ }^{8}$ - Yuan-Han Yang ${ }^{9,10}$
}

Published online: 13 February 2017

(C) The American Society for Experimental NeuroTherapeutics, Inc. 2017

\begin{abstract}
Whether antiplatelet agents have a preventive effect on cognitive function remains unknown. We examined the potential association between the use of cilostazol, an antiplatelet agent and cyclic adenosine monophosphate phosphodiesterase 3 inhibitor, and the risk of dementia in an Asian population. Patients initiating cilostazol therapy between 1 January 2004 and 31 December 2009 without a prior history of dementia were identified from Taiwan's National Health Insurance database. Participants were stratified by age, sex, comorbidities, and comedication. The outcome of interest was all-cause dementia (ICD-9CM codes 290.0, 290.4, 294.1, 331.0). Cox regression models were used to estimate the hazard ratio (HR) of dementia. The cumulative cilostazol dosage was stratified by quartile of defined daily doses using no cilostazol use as a reference. A total of 9148 participants 40 years of age or older and free of dementia at baseline were analyzed.
\end{abstract}

Yuan-Han Yang

endless@kmu.edu.tw

1 Department of Family Medicine, School of Medicine, College of Medicine, Kaohsiung Medical University, Kaohsiung City, Taiwan

2 Department of Family Medicine, Kaohsiung Medical University Hospital, Kaohsiung Medical University, Kaohsiung City, Taiwan

3 Department of Family Medicine, Kaohsiung Municipal Ta-Tung Hospital, Kaohsiung Medical University Hospital, Kaohsiung Medical University, Kaohsiung City, Taiwan

4 Research Center for Environmental Medicine, Kaohsiung Medical University, Kaohsiung City, Taiwan

5 Department of Otorhinolaryngology, School of Medicine, College of Medicine, Kaohsiung Medical University, Kaohsiung City, Taiwan
Patients using cilostazol $(n=2287)$ had a significantly decreased risk of incident dementia compared with patients not using the drug $[n=6861$; adjusted HR (aHR) 0.75; 95\% confidence interval (CI) 0.61-0.92]. Notably, cilostazol use was found to have a dose-dependent association with reduced rate of dementia emergence ( $p$ for trend $=0.001)$. Subgroup analysis identified a decline of dementia in cilostazol users with diagnosed ischemic heart disease (aHR 0.44, 95\% CI 0.24-0.83) and cerebral vascular disease (aHR 0.34, 95\% CI 0.21-0.54). These observations suggest that cilostazol use may reduce the risk to develop dementia, and a high cumulative dose further decreases the risk of dementia. These findings should be examined further in randomized clinical trials.

Key Words Dementia $\cdot$ cilostazol $\cdot$ cohort studies

6 Department of Otorhinolaryngology, Kaohsiung Medical University Hospital, Kaohsiung Medical University, Kaohsiung City, Taiwan

7 Department of Otorhinolaryngology, Kaohsiung Municipal Hsiao-Kang Hospital, Kaohsiung Medical University, Kaohsiung City, Taiwan

8 Management Offices, Kaohsiung Municipal Ta-Tung Hospital, Kaohsiung Medical University, Kaohsiung City, Taiwan

9 Department of Neurology, Kaohsiung Municipal Ta-Tung Hospital, Kaohsiung Medical University, Kaohsiung City, Taiwan

10 Department of Master's Program in Neurology, Faculty of Medicine, College of Medicine, Kaohsiung Medical University, Kaohsiung City, Taiwan 


\section{Introduction}

The proportion of elderly people in the total population of Taiwan has risen continually over the last 3 decades (from $4.1 \%$ in 1980 to $11.5 \%$ in 2013), and is estimated to reach $20 \%$ by 2025 [1], the highest rate of aging in the world [2]. With the rapid increase in the proportion of the elderly worldwide, the global prevalence of dementia is expected to increase dramatically by 2050 , with Asia estimated to increase by $59 \%$ [3]. There is currently no preventive or restorative treatment for loss of cognition culminating in dementia in the elderly $[4,5]$. Cognitive decline has been associated with the accumulation of $\beta$-amyloid $(A \beta)$ in brain parenchyma and vasculature, as well as cerebral ischemia. These 2 pathogenic mechanisms often coexist, and their contributions likely vary among different patients [6], making it difficult to determine causation. Therefore, an effective and optimal therapeutic strategy would ideally target both mechanisms by attenuating the accumulation of $A \beta$ and improving cerebral circulation, to ensure its metabolic needs are met.

Cilostazol is an antiplatelet drug that inhibits the activity of cyclic adenosine monophosphate (cAMP) phosphodiesterase type 3 and is prescribed clinically for the prevention of cerebral ischemia [7, 8]. It reportedly exerts a protective effect on endothelium and sustains blood flow via endotheliumindependent vasodilatation $[9,10]$. In addition, cilostazol has been shown to decrease accumulation of $A \beta$ and protect against $A \beta$-induced cognitive deficits in an experimental model [11].

This study investigated the potential risk-benefit ratio of using cilostazol to reduce risk of dementia in a large Asian population (Taiwan National Health Insurance Database). Comparisons were made for cumulative cilostazol usage among all patients without prior history of dementia. We hypothesized that cilostazol, a phosphodiesterase 3 inhibitor, might decrease the risk of all-cause dementia.

\section{Materials and methods}

\section{Data Source}

The present study was conducted using claims data from Taiwan's National Health Insurance Research Database (NHIRD), which is managed by the National Health Research Institute in Taiwan. The National Health Insurance (NHI) program reimburses healthcare costs of $99 \%$ of Taiwan's population (approximately 23 million people). The NHIRD contains comprehensive healthcare information for all of Taiwan's insured individuals, including demographic data, dates of clinical visits, diagnostic codes, and prescription details. For this study, we tapped a subset of NHIRD known as the Longitudinal Health Insurance Database (LHID) 2000.
This subset contains historical ambulatory and inpatient care data for 1 million randomly sampled beneficiaries enrolled into the NHI system in the year of 2000. The LHID 2000 database allows researchers to follow up the medical service utilization of these 1 million subjects. According to the National Health Research Institute, there are no statistically significant differences in age, sex, or healthcare costs between the LHID 2000 and NHIRD.

\section{Study Patients}

We identified patients who had ever taken cilostazol for at least 3 months between 1 January 2004 and 31 December 2009 for a cilostazol cohort. Cilostazol tablets are indicated for the reduction of symptoms of intermittent claudication in individuals with peripheral vascular diseases according to Taiwan's NHI guidelines. Clinicians usually start patients on cilostazol $50 \mathrm{mg}$ twice daily and then titrate this to full dose (100 mg twice daily). This cohort of cilostazol users was propensity matched with a comparison cohort, which consisted of patients who had never been treated with cilostazol, randomly sampled from the remaining subjects of the LHID 2000 data set and matched (1:3) on the basis of propensity score, including age, sex, index year, comorbidities, Charlson's index score, and comedications. The date of first use of cilostazol for each patient was defined as index date. To be included, no study subject could have been treated with any cilostazol therapy for 12 months prior to the first prescription (index date). Subjects were also excluded if they were under 40 years of age or over 90 years of age $(n=508,291)$, had all-cause dementia diagnosed before the index date $(n=4656)$, had a cumulative cilostazol usage duration of less than 3 months $(n=2635)$, or had incomplete follow-up insurance data $(n=737)$ (Fig. 1).

\section{Cumulative Exposure of Cilostazol}

Drug usage information was obtained from the outpatient pharmacy prescription database and included prescribed drug dosage, date of prescription, number of days supplied, and total number of pills dispensed. As patients might discontinue or restart drug therapy, the analyses assumed that patients' exposure to cilostazol contributed both cumulatively and continuously to their long-term risk of dementia. Defined daily dose (DDD) is the assumed average maintenance dose per day for a drug used for its main indication in adults [12]. To investigate the effect of dose, the cumulative use of cilostazol was calculated as total prescribed DDD (i.e., the same as total dispensed DDD under this system). The cumulative cilostazol dosage was calculated at the time a dementia event occurred and represents a total of DDD from drug initiation to the day before hospitalization for that event. For those who were still at risk (event-free and uncensored) in the cohort, the cumulative doses were recorded and ranked at each event. Participants were then 
Fig. 1 Study flowchart

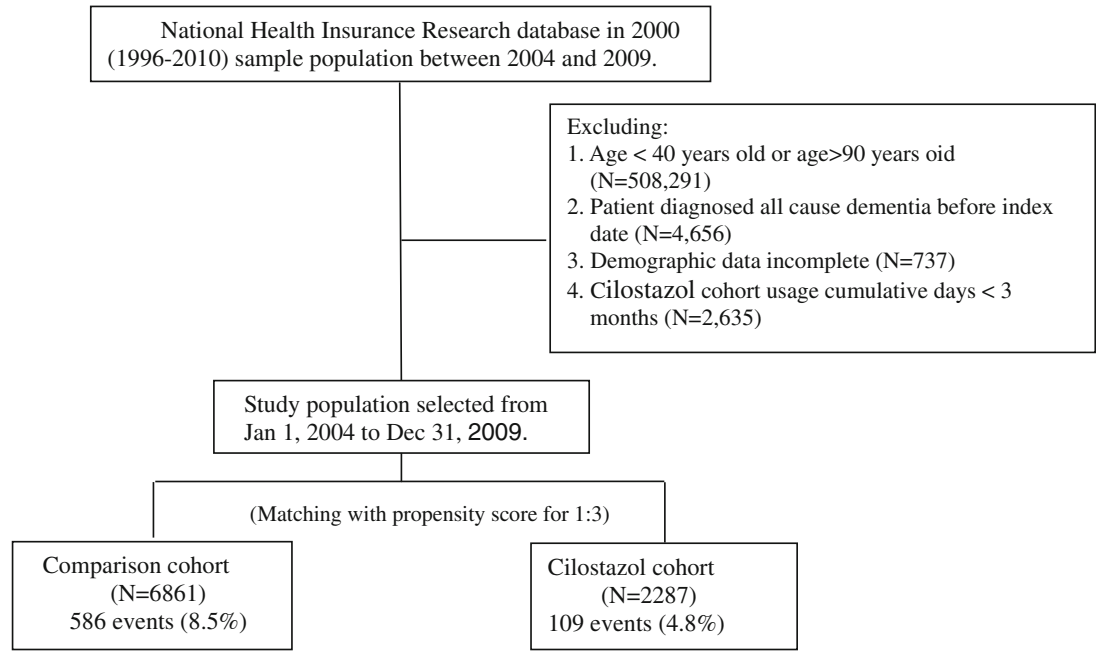

classified into mutually exclusive dosage categories based on the quartiles of cumulative dosage distribution [13]. As time passed and the accumulated cilostazol dosage changed during the follow-up period, a participant could be reassigned to either a higher or a lower quartile.

\section{Diagnosis of Dementia}

The outcome of interest was a diagnosis of ICD-9-CM, either 290.0 (Senile dementia, uncomplicated), 290.4x (Arteriosclerotic dementia), 294.1 (Dementia in conditions classified elsewhere), or 331.0 (Alzheimer disease). A diagnosis of dementia was based on at least 2 outpatient visits or at least 1 inpatient visit with a diagnosis of dementia listed on the submitted claims filing. Patients were followed from the index date to the earliest occurrence of dementia, death, disenrollment from the national health insurance program, or the end of the study date (31 December 2010), whichever came first. This study was approved by the Institutional Review Board of Kaohsiung Medical University Hospital (KMUHIRBEXEMPT (II)-20160028). Because the patient identifiers were scrambled before their data were used for research purposes to protect confidentiality, the requirement for written or verbal consent from patients for data linkage was waived.

\section{Covariates}

Inpatient and outpatient claim filings from the year prior to the index date were used to obtain information on comorbidities, including diabetes mellitus (ICD-9-CM:250.xx), hypertension (ICD-9-CM:401x), hyperlipidemia (ICD-9-CM:272x), peripheral vascular disease (ICD-9-CM:443), ischemic heart disease (ICD-9-CM: 433-438), depression (ICD-9-CM: 296.x-, 300.4, 311), ischemic stroke (ICD-9-CM:433-438), and Charlson's index score. Demographic data collected were age and sex. Comorbidities were defined in a patient if he or she was diagnosed for any of the aforementioned diseases on at least 2 outpatient claims or 1 inpatient claim during the exposure period. To control for confounding agents, we included drugs that could potentially accelerate or reduce inflammation or cognitive function in the model. These drugs were anticoagulants, antiplatelet agents, antidiabetic agents, antihypertensive agents, and statins. Exposure to these drugs was defined as having a prescription of one of them at least 1 day and calculated from the index date to the occurrence of any event related to this study, being disenrolled from the NHI program, death, or the end of the study period (31 December 2010), whichever came first.

\section{Statistical Analysis}

Pearson's $\chi^{2}$ test was performed to evaluate differences in categorical data between the cilostazol cohort and comparison cohort, including demographic data and comorbidities.

Cox proportional hazards regression analysis was performed to examine the risk of dementia in the cilostazol cohort versus the comparison cohort during the followup period. The DDDs recommended by the World Health Organization were used to quantify the use of cilostazol. Cumulative DDD was estimated as the sum of dispensed DDDs of cilostazol from 1 January 2004 to the date of dementia diagnosis or until the end of the study. The patients on cilostazol were categorized into low-dose (<86 DDDs), moderate-dose (86-217 DDDs), and high-dose ( $>217$ DDDs) users. Several covariables, including age, sex, comorbidities, and comedication, were adopted in the statistical analysis model. Hazard ratios (HR) and $95 \%$ confidence intervals (CIs), using the comparison cohort as the reference, were calculated to show the risk of dementia in patients on cilostazol 
Table 1 Demographic data for cilostazol cohort and comparison cohort $(n=9148)$

\begin{tabular}{|c|c|c|c|c|c|}
\hline \multirow[t]{2}{*}{ Variables } & \multicolumn{2}{|c|}{ Comparison cohort $(n=6861)$} & \multicolumn{2}{|c|}{ Cilostazol cohort $(n=2287)$} & \multirow[t]{2}{*}{$p$-value } \\
\hline & $n$ & $\%$ & $n$ & $\%$ & \\
\hline \multicolumn{6}{|l|}{ Age (years) } \\
\hline$<65$ & 2425 & 35.3 & 797 & 34.8 & \multirow[t]{2}{*}{0.667} \\
\hline$\geq 65$ & 4436 & 64.7 & 1490 & 65.2 & \\
\hline \multicolumn{6}{|l|}{ Sex } \\
\hline Female & 3274 & 47.7 & 1067 & 46.7 & \multirow[t]{2}{*}{0.378} \\
\hline Male & 3587 & 52.3 & 1220 & 53.3 & \\
\hline \multicolumn{6}{|l|}{ Comorbidities } \\
\hline Diabetes & 4047 & 59.0 & 1358 & 59.4 & 0.740 \\
\hline Hyperlipidemia & 3483 & 50.8 & 1174 & 51.3 & 0.638 \\
\hline Hypertension & 5540 & 80.7 & 1808 & 79.1 & 0.078 \\
\hline Depression & 967 & 14.1 & 296 & 12.9 & 0.167 \\
\hline Ischemic heart disease & 833 & 12.1 & 308 & 13.5 & 0.096 \\
\hline Cerebral vascular disease & 1416 & 20.6 & 478 & 20.9 & 0.789 \\
\hline \multicolumn{6}{|l|}{ Charlson's index score } \\
\hline$\leq 1$ & 2019 & 29.4 & 681 & 29.8 & \multirow[t]{3}{*}{0.696} \\
\hline 2 & 1409 & 20.5 & 484 & 21.2 & \\
\hline$\geq 3$ & 3433 & 50.0 & 1122 & 49.1 & \\
\hline \multicolumn{6}{|l|}{ Medication } \\
\hline Anticoagulant agents & 115 & 1.7 & 45 & 2.0 & 0.357 \\
\hline Antiplatetet agents & 1234 & 18.0 & 441 & 19.3 & 0.165 \\
\hline Antidiabetic agents & 3174 & 46.3 & 1046 & 45.7 & 0.663 \\
\hline Antihypertension agents & 4866 & 70.9 & 1595 & 69.7 & 0.283 \\
\hline Statin & 1529 & 22.3 & 539 & 23.6 & 0.204 \\
\hline
\end{tabular}

and in the dose-response analysis. Subgroup analysis was performed restricted to patients older than 60 years, who were more likely to develop dementia.

A Kaplan-Meier curve was used to estimate the probability of all-cause dementia, and log-rank test was used to evaluate the differences between with cilostazol cohort and comparison cohort. All statistical operations were performed using the SAS 9.3 statistical package (SAS Institute, Cary, NC, USA); all $p$-values were 2 -sided, and $p<0.05$ was considered significant.

\section{Results}

A total of 2287 patients initiating cilostazol from 1 January 2004 to 31 December 2009 were included (Fig. 1). After propensity score matching, 2287 cilostazol users were matched with 6861 noncilostazol users. As can be seen in Table 1, a summary of characteristics of cilostazol users and nonusers, all covariates were comparable after matching. In total, 109 $(4.8 \%)$ of cilostazol users, who were followed for an average of 3.3 years, developed all cause dementia; $586(8.5 \%)$ of
Table 2 The risk of all-cause dementia between cilostazol cohort and comparison cohort

\begin{tabular}{lllllll}
\hline & Case no. & $\%$ & cHR (95\% CI) & $\begin{array}{l}p \text { - } \\
\text { value }\end{array}$ & aHR (95\% CI) & $\begin{array}{l}p- \\
\text { value }\end{array}$ \\
\hline Comparison cohort $(n=6861)$ & 586 & 8.5 & Ref. & & Ref. & \\
Cilostazol cohort $(n=2287)$ & 109 & 4.8 & $0.77(0.63-0.95)$ & 0.015 & $0.75(0.61-0.92)$ & 0.006 \\
$<$ 86 DDDs $(\mathrm{N}=763)$ & 40 & 5.2 & $0.90(0.65-1.24)$ & 0.527 & $0.88(0.64-1.22)$ & 0.448 \\
86-217 DDDs $(\mathrm{N}=762)$ & 38 & 5.0 & $0.91(0.65-1.26)$ & 0.567 & $0.92(0.66-1.27)$ & 0.602 \\
$>$ 217 DDDs $(\mathrm{N}=762)$ & 31 & 4.1 & $0.57(0.40-0.82)$ & 0.002 & $0.53(0.37-0.76)$ & 0.001 \\
$p$ for trend & & & & 0.003 & & 0.001 \\
\hline
\end{tabular}

Adjusted for age, sex, all comorbidities, and history of medication. $\mathrm{cHR}=$ crude hazard ratio; $\mathrm{CI}=$ confidence interval; aHR $=$ adjusted hazard ratio; $\mathrm{DDD}=$ defined daily dose. 
a

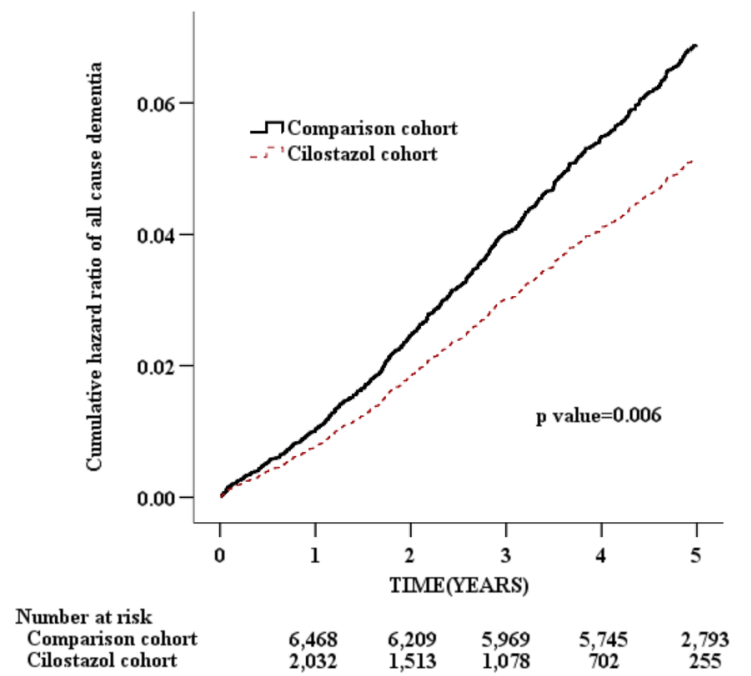

b

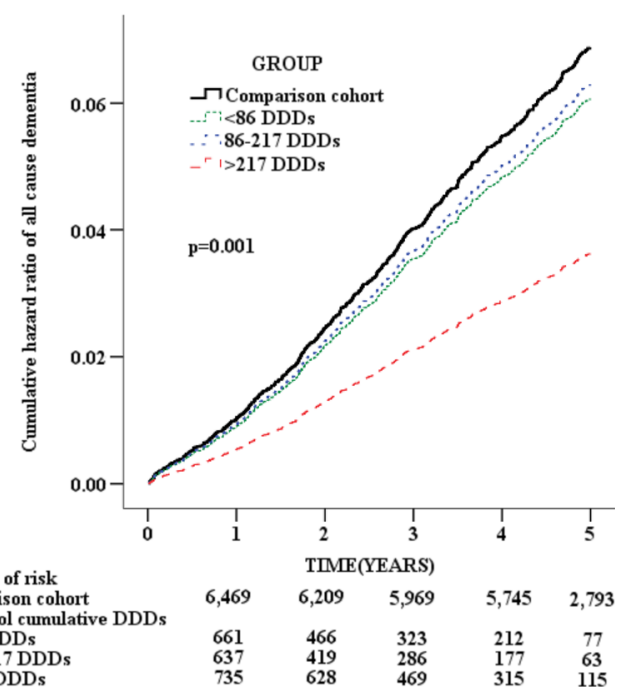

Fig. 2 All-cause dementia by (a) use and (b) defined daily doses (DDDs) of cilostazol

noncilostazol users, who were followed for an average of 4.5 years, developed all-cause dementia. Cox proportional hazard regression analysis revealed that cilostazol users were $23 \%$ less likely to develop dementia than the matched nonusers (crude HR 0.77, 95\% CI 0.63-0.95; $p=0.015$ ). The decreased risk of dementia remained significant after adjusting for potential confounders (adjusted HR 0.75, 95\% CI 0.61-0.92; $p=0.006$ ) (Table 2).

We further evaluated cilostazol as a dose-dependent factor for dementia by dividing the patients into 3 subgroups based on cumulative DDD of cilostazol (low dose: < 86 DDDs; moderate dose: 86-217 DDDs; high dose: > 217 DDDs). Incidence of dementia was $5.2 \%, 5.0 \%$, and $4.1 \%$ in the low-dose, moderate-dose, and high-dose groups, respectively. Their adjusted HRs for developing dementia were $0.88(95 \%$ CI $0.64-1.22 ; p=0.448), 0.92$ (95\% CI 0.66-1.27; $p=0.602)$, and 0.53 (95\% CI $0.37-0.76 ; p=0.001)$, respectively, after adjusting for potential confounders ( $p$ for trend $=0.001$ ) (Table 2). Based on these findings, high-dose cilostazol significantly and dose dependently decreased the risk of dementia. These findings were similar restricted to patients aged $\geq$ 60 years (Table S1; see Supplementary Material). Figure 2 illustrates all-cause dementia by use and DDD of cilostazol.

Compared with non-cilostazol users, male cilostazol users were at significantly lower risk of dementia (HR 0.58, 95\% CI 0.43-0.80), but female users were not (HR 0.91, 95\% CI $0.69-1.20)(p$-value for interaction $=0.039)($ Table 3$)$. In addition, cilostazol users over 65 years of age were at significantly lower risk of dementia (HR 0.69, 95\% CI 0.56-0.86), but those younger were not (HR 1.49, 95\% CI 0.81-2.76) ( $p$ value for interaction $=0.021)$. The effect of cilostazol use on dementia was significantly different among patients who had cerebral vascular disease and those who did not have that disease (HR 0.34, 95\% CI 0.21-0.54) ( $p$-value for interaction
$<0.001)$, but that effect did not differ significantly among those who had other comorbidities or not. In addition, regardless of whether cilostazol users were taking other medications or not, they had a lower risk of dementia than nonusers, although the difference between groups was not significant (Table 3).

\section{Discussion}

This study found a significant association between cilostazol use and a decreased risk of dementia among patients without prior history of dementia. We found a dose-response relationship between cilostazol use and risk of dementia. In addition, the effect was predominant among men aged older than 65 years with cerebral vascular disease. To the best of our knowledge, this is the first population-based, propensitymatched cohort study to explore the link between cilostazol use and the risk of dementia.

Cilostazol, a selective inhibitor of cyclic nucleotide phosphodiesterase 3 , has many pharmacological activities, including anti-inflammatory, antioxidative, and antiapoptotic effects in the brain [14]. It has been found to be beneficial in the treatment of ischemic heart disease and poststroke patients [15-17]. In addition, cilostazol has been found to prevent cerebral hypoperfusion-induced cognitive impairment and white matter damage induced by occluding the common carotid artery by bilateral ligation in rats [18]. In a recent translational overview, phosphodiesterase inhibitors were reported to enhance cAMP and/or cyclic guanosine monophosphate signaling by reducing the degradation of these cyclic nucleotides [19]. As both cAMP and cyclic guanosine monophosphate signaling are essential to a variety of cellular functions, including neuroplasticity and neuroprotection, 
Table 3 The risk of all-cause dementia between cilostazol cohort and comparison cohort split by comorbidities $(n=9148)$

\begin{tabular}{|c|c|c|c|c|c|c|c|}
\hline & \multicolumn{2}{|c|}{ Comparison cohort } & \multicolumn{2}{|c|}{ Cilostazol cohort } & \multicolumn{2}{|c|}{ Cilostazol cohort $v s$ comparison cohort } & \multirow{2}{*}{$\begin{array}{l}\text { Interaction } \\
p \text {-value }\end{array}$} \\
\hline & No. cases & $\%$ & No. cases & $\%$ & Adjusted HR (95\% CI) & $p$-value & \\
\hline \multicolumn{8}{|l|}{ Sex } \\
\hline Female & 290 & 8.9 & 64 & 6.0 & $0.91(0.69-1.20)$ & 0.505 & \multirow[t]{2}{*}{0.039} \\
\hline Male & 296 & 8.3 & 45 & 3.7 & $0.58(0.43-0.80)$ & 0.001 & \\
\hline \multicolumn{8}{|c|}{ Age (years) } \\
\hline$<65$ & 42 & 1.7 & 14 & 1.8 & $1.49(0.81-2.76)$ & 0.199 & \multirow[t]{2}{*}{0.021} \\
\hline$\geq 65$ & 544 & 12.3 & 95 & 6.4 & $0.69(0.56-0.86)$ & 0.001 & \\
\hline \multicolumn{8}{|c|}{ Comorbidities } \\
\hline \multicolumn{8}{|l|}{ Diabetes } \\
\hline No & 239 & 8.5 & 46 & 5.0 & $0.76(0.55-1.05)$ & 0.093 & \multirow[t]{2}{*}{0.709} \\
\hline Yes & 347 & 8.6 & 63 & 4.6 & $0.74(0.56-0.96)$ & 0.026 & \\
\hline \multicolumn{8}{|c|}{ Hyperlipidemia } \\
\hline No & 332 & 9.8 & 56 & 5.0 & $0.64(0.48-0.85)$ & 0.002 & \multirow[t]{2}{*}{0.191} \\
\hline Yes & 254 & 7.3 & 53 & 4.5 & $0.91(0.67-1.22)$ & 0.525 & \\
\hline \multicolumn{8}{|c|}{ Hypertension } \\
\hline No & 74 & 5.6 & 14 & 2.9 & $0.63(0.35-1.11)$ & 0.110 & \multirow[t]{2}{*}{0.492} \\
\hline Yes & 512 & 9.2 & 95 & 5.3 & $0.77(0.62-0.96)$ & 0.022 & \\
\hline \multicolumn{8}{|c|}{ Depression } \\
\hline No & 477 & 8.1 & 89 & 4.5 & $0.75(0.60-0.94)$ & 0.012 & \multirow[t]{2}{*}{0.876} \\
\hline Yes & 109 & 11.3 & 20 & 6.8 & $0.76(0.47-1.22)$ & 0.252 & \\
\hline \multicolumn{8}{|c|}{ Ischemic heart disease } \\
\hline No & 490 & 8.1 & 98 & 5.0 & $0.81(0.65-1.00)$ & 0.055 & \multirow[t]{2}{*}{0.125} \\
\hline Yes & 96 & 11.5 & 11 & 3.6 & $0.44(0.24-0.83)$ & 0.011 & \\
\hline \multicolumn{8}{|c|}{ Cerebral vascular disease } \\
\hline No & 382 & 7.0 & 89 & 4.9 & $1.01(0.80-1.28)$ & 0.932 & \multirow[t]{2}{*}{$<0.001$} \\
\hline Yes & 204 & 14.4 & 20 & 4.2 & $0.34(0.21-0.54)$ & $<0.001$ & \\
\hline \multicolumn{8}{|l|}{ Medication } \\
\hline \multicolumn{8}{|c|}{ Anticoagulant agents } \\
\hline No & 565 & 8.4 & 106 & 4.7 & $0.76(0.62-0.94)$ & 0.010 & \multirow[t]{2}{*}{0.576} \\
\hline Yes & 21 & 18.3 & 3 & 6.7 & $0.31(0.09-1.12)$ & 0.074 & \\
\hline Antiplat & et agents & & & & & & \\
\hline No & 465 & 8.3 & 87 & 4.7 & $0.77(0.61-0.97)$ & 0.026 & 0.528 \\
\hline Yes & 121 & 9.8 & 22 & 5.0 & $0.66(0.42-1.04)$ & 0.073 & \\
\hline Antidiab & tic agents & & & & & & \\
\hline No & 323 & 8.8 & 63 & 5.1 & $0.75(0.57-0.99)$ & 0.040 & 0.742 \\
\hline Yes & 263 & 8.3 & 46 & 4.4 & $0.74(0.54-1.02)$ & 0.063 & \\
\hline Antihyp & tension age & & & & & & \\
\hline No & 145 & 7.3 & 23 & 3.3 & $0.57(0.36-0.88)$ & 0.012 & 0.253 \\
\hline Yes & 441 & 9.1 & 86 & 5.4 & $0.82(0.65-1.04)$ & 0.094 & \\
\hline Statin & & & & & & & \\
\hline No & 482 & 9.0 & 88 & 5.0 & $0.93(0.58-0.92)$ & 0.007 & 0.798 \\
\hline Yes & 104 & 6.8 & 21 & 3.9 & $0.86(0.54-1.39)$ & 0.544 & \\
\hline
\end{tabular}

Adjusted for age, sex, comorbidities, and history of medication. Values in bold are significant. HR = hazard ratio; $\mathrm{CI}=$ confidence interval
Phosphodiesterase inhibitors are receiving increased research attention for their potential use in the treatment age-related cognitive decline, as well as dementia in animals and humans $[19,20]$. Cilostazol could potentially be used to prevent neuronal death and cognitive impairment as it has been found to exert a protective effect on cognitive decline in patients with cerebral infarctions [21], and in rat models of chronic cerebral hypoperfusion [22-24]. One possible mechanism for its effect 
may be increased cerebral blood flow and the subsequent prevention of demyelination or microinfarction of the cerebral white matter, though this has not been observed in neuroimaging studies in either rats with chronic cerebral hypoperfusion [23], or patients with Alzheimer's disease (AD) [25]. One retrospective study reported that cilostazol improved cognitive function in patients with mild cognitive impairment [26]. A pilot study of 10 patients with moderate AD conducted in a clinical setting reported that cilostazol slowed the trajectory of cognitive decline when coadministered with donepezil after an average follow-up period of 9.4 months [20]. It should also be noted that cilostazol is being investigated in a clinical phase IV study examining the effects of cilostazol augmentation in patients with mild-to-moderate $\mathrm{AD}$ in whom subcortical white matter hyperintensities were treated with the acetylcholinesterase inhibitor donepezil [27]. It has also been reported to suppress effectively cognitive decline in patients with mild dementia receiving donepezil, but not in those with moderate and severe dementia [28].

This study also found the effect of cilostazol use on the risk of dementia to be notable among patients with ischemic heart disease (56\% risk reduction) and those with cerebral vascular disease (66\% risk reduction). Sakurai et al. [29] conducted a pilot study with a sample of 20 patients with mild-to-moderate $\mathrm{AD}$ and cerebrovascular disease who received daily cilostazol $100 \mathrm{~g}(n=11)$, or daily clopidogrel or aspirin $(n=9)$ for 6 months. The patients with cilostazol treatment showed no further cognitive decline, while those with clopidogrel or aspirin treat did. The effect of cilostazol on dementia may come about through its improvement in blood flow, though further studies are needed to confirm this possibility. However, despite the positive effect of cilostazol on dementia, the Food and Drug Administration has announced that patients with class III-IV heart failure who use cilostazol, inhibitors of phosphodiesterase 3 , and several of its metabolites, do not survive as long as the same type of patients taking a placebo [30]. Thus, future studies should focus on both its efficacy and safety.

This study has some limitations. It is limited by the possibility of residual confounding and the potential for confounding by indication on claims files. To minimize the inherent bias of the observational study, we identified the study drug exposure and comorbidities of the patients before the index date and propensity matched them to form a comparison group. This would increase comparability of both study groups. Another limitation is that this study is based on claims data that do not include data on other risk factors for dementia (e.g., ApoE4 allele carrier, educational level, or lifestyle factors). Another limitation is that we were not able to differentiate between different etiologies of dementia, such as the dementia that occurs during the course of $\mathrm{AD}$ or vascular dementia. Thus, there was a high rate of diagnoses of unspecified and mixed dementia among the claims filings.
In conclusion, this prospective cohort study found that cilostazol, which affects both cerebral circulation and $\mathrm{A} \beta$ metabolism, may reduce the risk of dementia, a disease for which no treatments currently exist. Our results highlight the need for large-scale, double-blind, long-term studies to clarify the therapeutic response of cilostazol on the preservation of cognitive function in patients with dementia.

Acknowledgments This study was supported by grants from Kaohsiung Medical University Hospital (KMUH103-3R68 and KMUH105-5R64), the Ministry of Science and Technology (MOST103-2314-B-037-004-MY3), and Research Center for Environmental Medicine, Kaohsiung Medical University (KMUTP104A34), none of which have had any role in study design, data collection and analysis, decision to publish, or preparation of the manuscript. The authors are grateful for the assistance of the Statistical Analysis Laboratory, Department of Medical Research, Kaohsiung Municipal TaTung Hospital.

Required Author Forms Disclosure forms provided by the authors are available with the online version of this article.

Compliance with Ethical Standards

Conflict of Interest The authors have no conflict of interest to declare.

\section{References}

1. Department of Statistics, Ministry of the Interior, Taiwan, [online]. Available at: http://www.moi.gov.tw/stat/. Accessed January 25, 2017.

2. The China Post news staff. Taiwan must address the challenges of an aging society [online]. Available at: http://www.chinapost.com. tw/editorial/taiwan-issues/2013/10/13/391104/Taiwan-must.htm. Accessed October 13, 2013.

3. Brookmeyer R, Johnson E, Ziegler-Graham K, Arrighi HM. Forecasting the global burden of Alzheimer's disease. Alzheimers Dement. 2007;3(3):186-91.

4. Drugs for Alzheimer's disease: best avoided. No therapeutic advantage. Prescrire international. 2012;21(128):150.

5. Birks J, Flicker L. Donepezil for mild cognitive impairment. The Cochrane database of systematic reviews. 2006(3):CD006104.

6. Taguchi A. Vascular factors in diabetes and Alzheimer's disease. J Alzheimers Dis. 2009;16(4):859-64.

7. Gresele P, Momi S, Falcinelli E. Anti-platelet therapy: phosphodiesterase inhibitors. British Journal of Clinical Pharmacology. 2011;72(4):634-46.

8. Shinohara Y, Katayama Y, Uchiyama S, Yamaguchi T, Handa S, Matsuoka K, et al. Cilostazol for prevention of secondary stroke (CSPS 2): an aspirin-controlled, double-blind, randomised non-inferiority trial. The Lancet Neurology. 2010;9(10):959-68.

9. Kasahara Y, Nakagomi T, Matsuyama T, Stern D, Taguchi A. Cilostazol Reduces the Risk of Hemorrhagic Infarction After Administration of Tissue-Type Plasminogen Activator in a Murine Stroke Model. Stroke. 2012;43(2):499-506.

10. Tanaka K, Gotoh F, Fukuuchi Y, Amano T, Uematsu D, Kawamura $\mathrm{J}$, et al. Effects of a selective inhibitor of cyclic AMP phosphodiesterase on the pial microcirculation in feline cerebral ischemia. Stroke. 1989;20(5):668-73.

11. Park SH, Kim JH, Bae SS, Hong KW, Lee DS, Leem JY, et al. Protective effect of the phosphodiesterase III inhibitor cilostazol 
on amyloid beta-induced cognitive deficits associated with decreased amyloid beta accumulation. Biochem Biophys Res Commun. 2011;408(4):602-8.

12. WHO Collaborating Centre for Drug Statistics Methodology. Defined daily dose: definition and general considerations [online]. Available at: https://www.whocc.no/ddd/definition_and_general_ considera/. Accessed December 20, 2016.

13. Stricker BH, Stijnen T. Analysis of individual drug use as a timevarying determinant of exposure in prospective population-based cohort studies. Eur J Epidemiol. 2010;25(4):245-51.

14. Hong KW, Lee JH, Kima KY, Park SY, Lee WS. Cilostazol: therapeutic potential against focal cerebral ischemic damage. Curr Pharm Des. 2006;12(5):565-73.

15. Zheng XT, Chen KY, Liu T, Xu LX, Che JJ, Rha SW, et al. Lowdose adjunctive cilostazol in patients with complex lesions undergoing percutaneous coronary intervention. Clin Exp Pharmacol Physiol. 2016;43(1):29-33.

16. Wang W, Zhang L, Liu W, Zhu Q, Lan Q, Zhao J. Antiplatelet Agents for the Secondary Prevention of Ischemic Stroke or Transient Ischemic Attack: A Network Meta-Analysis. Journal of stroke and cerebrovascular diseases : the official journal of National Stroke Association. 2016;25(5):1081-9.

17. Ueda H, Kido A, Matsuhisa S, Asawa K, Yoshida N, Tsujimoto M, et al. Addition of cilostazol to aspirin therapy for secondary prevention of cardiovascular and cerebrovascular disease in patients undergoing percutaneous coronary intervention: A randomized, openlabel trial. Am Heart J. 2016;173:134-42.

18. Lee JH, Park SY, Shin YW, Hong KW, Kim CD, Sung SM, et al. Neuroprotection by cilostazol, a phosphodiesterase type 3 inhibitor, against apoptotic white matter changes in rat after chronic cerebral hypoperfusion. Brain research. 2006;1082(1):182-91.

19. Heckman PR, Wouters C, Prickaerts J. Phosphodiesterase inhibitors as a target for cognition enhancement in aging and Alzheimer's disease: a translational overview. Curr Pharm Des. 2015;21(3): 317-31.

20. Arai H, Takahashi T. A combination therapy of donepezil and cilostazol for patients with moderate Alzheimer disease: pilot follow-up study. Am J Geriatr Psychiatry. 2009;17(4):353-4.

21. Mochizuki Y, Oishi M, Mizutani T. Effects of cilostazol on cerebral blood flow, P300, and serum lipid levels in the chronic stage of cerebral infarction. Journal of stroke and cerebrovascular diseases : the official journal of National Stroke Association. 2001;10(2):639.

22. Puzzo D, Vitolo O, Trinchese F, Jacob JP, Palmeri A, Arancio O. Amyloid-beta peptide inhibits activation of the nitric oxide/cGMP/ cAMP-responsive element-binding protein pathway during hippocampal synaptic plasticity. The Journal of neuroscience : the official journal of the Society for Neuroscience. 2005;25(29):6887-97.

23. Watanabe T, Zhang N, Liu M, Tanaka R, Mizuno Y, Urabe T. Cilostazol protects against brain white matter damage and cognitive impairment in a rat model of chronic cerebral hypoperfusion. Stroke. 2006;37(6):1539-45.

24. Lee JH, Park SY, Shin YW, Kim CD, Lee WS, Hong KW. Concurrent administration of cilostazol with donepezil effectively improves cognitive dysfunction with increased neuroprotection after chronic cerebral hypoperfusion in rats. Brain research. 2007; 1185:246-55.

25. Bellew KM, Pigeon JG, Stang PE, Fleischman W, Gardner RM, Baker WW. Hypertension and the rate of cognitive decline in patients with dementia of the Alzheimer type. Alzheimer disease and associated disorders. 2004;18(4):208-13.

26. Taguchi A, Takata Y, Ihara M, Kasahara Y, Tsuji M, Nishino M, et al. Cilostazol improves cognitive function in patients with mild cognitive impairment: a retrospective analysis. Psychogeriatrics. 2013;13(3):164-9.

27. ClinicalTrials.gov [Internet]. Bethesda (MD): National Library of Medicine (US). Available from: http://clinicaltrials. gov/show/NCT01409564 (NLM Identifier: NCT01409564). Accessed July 12, 2016.

28. Ihara M, Nishino M, Taguchi A, Yamamoto Y, Hattori Y, Saito S, et al. Cilostazol add-on therapy in patients with mild dementia receiving donepezil: a retrospective study. PLoS One. 2014;9(2):e89516.

29. Sakurai H, Hanyu H, Sato T, Kume K, Hirao K, Kanetaka H, et al. Effects of cilostazol on cognition and regional cerebral blood flow in patients with Alzheimer's disease and cerebrovascular disease: a pilot study. Geriatr Gerontol Int. 2013;13(1):90-7.

30. Chi Y-W, Lavie CJ, Milani RV, White CJ. Safety and efficacy of cilostazol in the management of intermittent claudication. Vascular Health and Risk Management. 2008;4(6):1197-203. 